2 pg. whereas another. Bromus erectus, contains $22 \mathrm{pg}$ per nucleus. may explain the way in which these species partition the environmental resources and thereby manage to coexist. Studies of this kind will assist botanists to appreciate more fully the implications of Gause's competitive exclusion hypothesis in the realm of vegetation.
Al-Mufti M M. Sydes, C. L. Furness S. B., Grime. J.P. \& Band. S. R. J. Ecol. 65.759 (1977)

2. Duffey. E. Grassima Ecology and Wildife Managemen (Chapman and Hatl. London, 1974).

3rime. J.P.. Shacklock. J.M.L. \& Band, S.R. Nen Phitol. 100. +35 (1985)

. Grime. J.P. \& Mowforth, M.A. Nature 299, 151 (1982).

Peter D. Moore is in the Department of Plan Sciences, Universitv of London King's College. 68 Half Moon Lane, London SE24 9JF, UK

\title{
Astrophysics
}

\section{Phase transitions in star formation}

\section{from Alan P. Boss}

MANY dynamical aspects of star formation have become understood through theoretical models of collapsing interstellar clouds but attention has recently been turned to equilibrium models. From models of the possible equilibrium states available to interstellar clouds and their protostellar progeny, it emerges that two types of equilibrium may occur for isothermal clouds with small angular momentum, with one being much denser than the other. Therefore, if there are phase transitions - mathematically analogous to the transition of a Van der Waals gas to a liquid - from the more diffuse to the more compact state, the result could be star formation in clouds that would otherwise be considered stable ${ }^{t}$.

An interstellar cloud must contract and increase its density by a factor of about $10^{21}$ in order to form a star. Observation has yielded little knowledge about the contraction process, because of the inherent obscuration and small length scales involved. Theoretical models of nonmagnetic star formation have focused on solving the exceedingly difficult time-dependent problem of the collapse of an interstellar cloud from an initially unstable state.

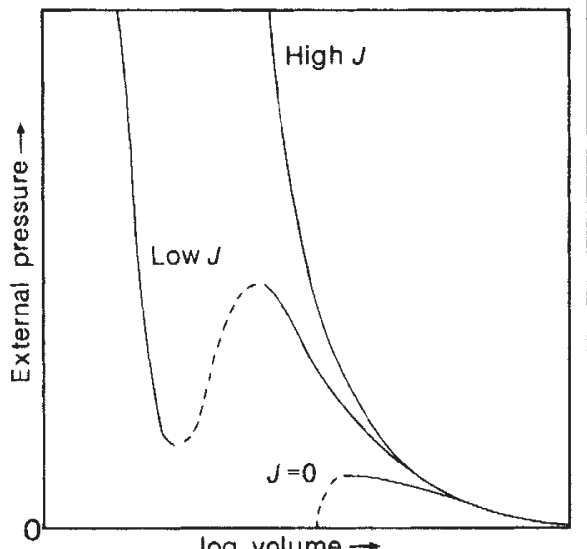

$\log$ volume $\rightarrow$

Axisymmetrical equilibrium sequences for isothermal clouds with differing amounts of angular momentum, $J$. No equilibrium exists for sufficiently dense clouds with $J=0$. Clouds with low $J$ can exist in either a diffuse or compact state, separated by unstable equilibria (broken line). For high $J$ clouds the unstable branch disappears. Phase transitions from the diffuse to the compact branch may initiate star formation (modified from ref. 2).
To include rotation and the possibility of fragmentation, the model must include all three spatial dimensions, a formidable task. Protostellar clouds are effectively isothermal during much of their evolution, but eventually they become opaque and heat up, adding further to the complexity of the computations.

A simpler approach is to seek equilibrium states for rotating, isothermal, axisymmetrical, self-gravitating clouds (see figure). Models based on the global properties of spheroids of uniform density reveal that, for clouds with low angular momentum, two types of equilibrium are possible ${ }^{2-4}$ : slowly-rotating, nearlyspherical clouds, supported primarily by external pressure (diffuse branch), and rapidly rotating, highly flattened clouds, supported largely by rotation (compact branch). Intermediate configurations are unstable to compression. For clouds with large angular momentum, a single sequence exists, extending monotonically to high densities.

The diffuse state is metastable, because its free energy is higher than the compact branch. The unstable branch has the highest free energy, forming a barrier between the stable branches. If the free energy of a diffuse branch cloud is raised sufficiently, such as by compression in the shock front associated with the spiral arms of our Galaxy, an otherwise stable cloud could dynamically become a compact cloud. Such a phase transition would allow low angular momentum clouds that are stable against collapse to become significantly denser ${ }^{1}$.

Phase transitions can only be considered physically possible if detailed models of the two branches can be constructed and shown to be dynamically stable. Rigorous modelling of clouds with external pressure produces stable clouds that become progressively more condensed at their centres as the mass increases ${ }^{5}$. The models become unstable to compression at high central density, as in non-rotating, isothermal clouds supported by external pressure. These models are the realistic manifestation of the diffuse branch ${ }^{3}$.

Analytical solutions exist ${ }^{6.7}$. They are compressionally unstable when the cloud is nearly spherical, and dynamically un- stable to ring formation when highly flattened $^{\text {n.s. }}$; intermediate models are stable to axisymmetrical perturbations. However, these solutions are mathematically singular at the origin and extend to infinity. Because of the lack of external pressure, the flattened solutions are similar to the compact branch. Thin-disk models have been constructed which also suffer from an infinite central density, but they do include external pressure". These models are the closest yet developed to a rigorous description of the compact branch. Because compact branch models with finite central density have not yet been constructed, and their stability to axisymmetrical perturbations has not been tested, it is premature to declare the compact branch a possible member of the physical universe. It is important to note that calculations of the dynamical collapse of isothermal clouds have found the diffuse branch" but not the compact branch. Instead, collapsing axisymmetrical clouds produce either rings or runaway disks, depending on details such as the initial distribution of angular momentum.

This discussion has dealt with stability to compressional or axisymmetrical perturbations but interstellar clouds can also distort in a non-axisymmetrical way. Simplified ellipsoidal models form a high angular momentum sequence (similar to the diffuse branch) that terminates when sufficiently compressed ${ }^{2}$. The simplified compact branch models are usually dynamically unstable to non-axisymmetrical distortions which could result in fragmentation and further contraction to the stellar state'. The stability of detailed models of the compact state to nonaxisymmetrical distortion is unknown.

If phase transitions occur between diffuse and compact interstellar clouds, it may be easier than has been supposed for the process of star formation to be initiated. Clouds that are stable to small perturbations may be hammered into a dynamic collapse towards a denser configuration. A collapsing cloud may well bypass any compact equilibrium state and fragment directly into a protostellar system. The validity of these speculations awaits confirmation of the existence and stability of the compact branch. Bear in mind, however, that unstable equilibrium states are of considerably less interest to nature than to theoretical astrophysicists.

\footnotetext{
1. Tohline. J.E. Astrophys. J. 292. 181 (1985).

2. Weber, S.V. Astrophys. J. 208,113 (1976)

3. Hachisu. 1. \& Eriguchi. Y. Astron. Astrophys. 140, 259 1984).

Tohline. J.E. Icarus 61, 10 (1985)

5. Stahler. S. W. Astrophvs. J. 268, 165 (1983).

Hayashi, C. et al. Prog. theor. Phys. 68. 1949 (1982)

6. Hayashi, C. et al. Prog. theor. Phys. 68. 1949

8. Toomre, A. Astrophys. J. 259. $535(1982)$.

8. Schmitz. F. Astron. Astrophys. 131. 3(4) (1984).
9. Hachisu. I. \& Eriguchi. Y. Astron. Astrophys, 143, 355 (1985)

10. Boss. A.P. \& Haber. J.G. Astrophys. J. 255. 240 (1982)
}

Alan P. Boss is in the Department of Terrestrial Magnetism, Carnegie Institution of Washington, 5241 Broad Branch Road NW, Washington DC 20015, USA. 